\title{
Does the dynamics of sine-Gordon solitons predict active regions of DNA?
}

\author{
Sara Cuenda ${ }^{\mathrm{a}, *}$, Angel Sánchez ${ }^{\mathrm{a}, \mathrm{b}}$, Niurka R. Quintero ${ }^{\mathrm{c}}$

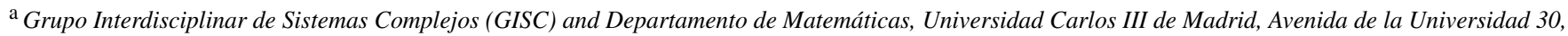 \\ 28911 Leganés, Madrid, Spain \\ ${ }^{\mathrm{b}}$ Instituto de Biocomputación y Física de Sistemas Complejos (BIFI), Universidad de Zaragoza, 50009 Zaragoza, Spain \\ ${ }^{\mathrm{c}}$ Universidad de Sevilla, Departamento de Física Aplicada I, E.U.P., Virgen de África 7, 41011 Sevilla, Spain
}

Received 19 June 2006; received in revised form 6 September 2006; accepted 16 September 2006

Available online 25 October 2006

Communicated by A. Mikhailov

\begin{abstract}
In this work we analyze the possibility that the soliton dynamics in a simple nonlinear model allows functionally relevant predictions of the behaviour of DNA. This suggestion was first put forward by Salerno [M. Salerno, Phys. Rev. A 44 (1991) 5292] by showing results indicating that sine-Gordon kinks were set in motion at certain regions of a DNA sequence that include promoters. We revisit that system and show that the observed behaviour has nothing to do with promoters; on the contrary, it originates from the bases at the boundary, which are not part of the genome studied. We explain this phenomenology in terms of an effective potential for the kink center. This is further extended to disprove recent claims that the dynamics of kinks [E. Lennholm, M. Hörnquist, Physica D 177 (2003) 233] or breathers [J.D. Bashford, J. Biol. Phys. 32 (2006) 27] has functional significance. We conclude that no such information can be extracted from this simple nonlinear model or its associated effective potential.
\end{abstract}

Keywords: DNA; Genome; Nonlinear dynamics; Solitons; Collective coordinates

\section{Introduction}

Nonlinear models supporting coherent excitations have appeared in many fields of science since the pioneering discoveries by Fermi, Pasta et al. [1] more than 50 years ago. The success of this approach in modeling complex systems has encouraged its application in other fields. That is the case for biology, where nonlinear models were widely applied in many subjects, such as in the study of the DNA molecule (see, for example, [2-4]). To realize the relevance of these models it should be noticed that, nowadays, the computational cost of molecular dynamics for realistic models of DNA molecules with a few tens of base pairs allows simulation times up to tens of nanoseconds at most. Nonlinear models allow the study of such a complex system with very many degrees of freedom by drastically reducing this amount up

\footnotetext{
* Corresponding author.

E-mail addresses: scuenda@math.uc3m.es (S. Cuenda), anxo@math.uc3m.es (A. Sánchez), niurka@euler.us.es (N.R. Quintero).
}

to one degree of freedom per base pair, the most relevant for the process under study. It goes without saying that the reduction of a very complicated object such as the DNA duplex to a polymer formed by base pairs, each one with just one degree of freedom (sometimes a few more), helps enormously in the theoretical and computational study of these models. Nevertheless, although simplified, these models can yield important results. An example of these models is the Peyrard-Bishop model of DNA [5], which achieved an important goal when describing the denaturation process of DNA in terms of just the radial distance of the bases on each base pair [6].

Among all these approaches we focus here on the work of Englander et al. [7], who introduced the sine-Gordon (sG) equation as a model for DNA in 1980. The existence of sG solitons in the DNA molecule has been surrounded by controversy, as expected in a field were biology and physics do not always meet in a fruitful way $[8,9]$. When Englander and co-workers introduced the sG model of DNA, they based their hypothesis on experimental results that showed unexpectedly 
long lifetimes of open states of DNA duplexes [10]. In spite of the fact that, later, Guéron et al. [11] found more reasonable lifetimes, smaller by one or two orders of magnitude than the ones reported in previous works, a vast amount of literature is still based on the Englander model. On the other hand, the very existence of solitons in DNA is questionable, as the viscous critical force of water is about a thousand times larger than the typical scale of forces in DNA (piconewton range). In fact, the effect of water friction damps out any inertial effect in the world of the cell and, consequently, in DNA [12]. In particular, this raises questions about the applicability of the sine-Gordon model of Englander et al. and related ones, which contain an inertial term at least of the order of the dissipative one: Dominance of the dissipative term would lead to pinning of sine-Gordon solitons and to annihilation of sine-Gordon breathers. Therefore, we want to stress that the approach we are dealing with here, namely DNA models with soliton-like excitations, is purely phenomenological and does not imply any claim concerning the true existence and character of such excitations.

In this context, and keeping in mind the above caveats, the aim of this work is to analyze in depth part of the literature related to the work of Englander et al., providing new results that give insight into a number of important questions. Specifically, we will study the relation between the dynamics of $\mathrm{sG}$ solitons and the position of promoters in the genome of the bacteriophage $T 7$. This line of work began with Salerno [13-15] at the beginning of the 1990s and was subsequently continued in several works [16-20]. We stress that this is a very important issue: Indeed, if the Englander model behaviour could be connected to functionally relevant positions in the sequence, it would provide a cheap and efficient tool for genomics. Claims in this direction have already been presented [20]. Note that the serious doubts about the existence of solitons in DNA discussed above would have no consequences for this application of the model, because nonlinear models might somehow phenomenologically correlate with important genomic features of sequences. However, as we will show below, the main result of the present work is that, unfortunately, such a connection cannot be substantiated for reasons intrinsic to the nonlinear models themselves.

The structure of the paper is as follows. In Section 2 we discuss the methodology and the results of the first two papers concerning this issue $[13,14]$ in terms of the effective potential introduced by Salerno in collaboration with Kivshar in [15]. In Section 3 we describe the main features of the promoters of the $T 7$ genome, and analyze the simulation results of the work of Lennholm and Hörnquist [17] in terms of the effective potential. In Section 4 we discuss recent work concerning breathers in the sG model [20]. Finally, Section 5 concludes the paper by summarizing our main results and their implications.

\section{Early work on $T 7: A_{1}, A_{0}$ and $A_{3}$ promoters}

More than a quarter of a century ago, Englander and coworkers [7] introduced solitonic excitations into the DNA world as an initial step towards understanding the stability of open segments of DNA molecules [10]. They suggested the well known sG model, that describes the dynamics of a line of pendula in a vertical gravitational field with torsional spring coupling between units, as an effective description of DNA molecules. In this way, the double helix is approximated by two parallel rods on which pendula (base pairs) are attached, and bonding to the opposite base is represented by a "gravitational" potential of each pendulum. Calling $\phi_{i}$ the twist angle of the $i$ th base, this model has static soliton (kink) solutions given by

$\phi_{i}=4 \arctan \left(\mathrm{e}^{a i}\right)$,

valid for $a \ll 1$, where the continuum approximation applies. In Eq. (1), $a$ is a dimensionless parameter representing the parameters of the model, and acts as an effective discretization parameter of the continuum $\mathrm{sG}$ problem. In spite of such a great oversimplification of the real problem, the model contained the main feature of breaking a bond around $\phi=0$. In addition to this, the results were consistent with available data [10] although Englander et al. were aware of the lack of evidence of solitonic excitations.

Salerno, in his pioneering and interesting work [13], tried to find a relation between relevant sites in the $T 7$ genome and the dynamics of sG kinks moving along the inhomogeneous DNA sequence under study; the main difference with respect to previous works was the introduction of the inhomogeneity of the sequence in the model. To do so, he took the static kink solution (1), with center at $n_{0}$, and used it as initial condition of the equations of motion of the discrete, inhomogeneous sG (or Englander) model,

$\ddot{\phi}_{i}=\phi_{i+1}-2 \phi_{i}+\phi_{i-1}-q_{i} \sin \phi_{i}$,

$q_{i}$ being the parameter that carries all the information of the sequence under study. It is defined as $q_{i}=\beta \lambda_{i} / K$, where $K$ is the torsional spring constant between consecutive bases, $\beta$ is the energy of a hydrogen bond and $\lambda_{i}$ is the number of hydrogen bonds in a base pair, which is $\lambda_{i}=2$ for $A T$ base pairs and $\lambda_{i}=3$ for $C G$ base pairs. Considered as a discrete version of the continuum $\mathrm{sG}$ equation, the effective discretization of the lattice used in [13] was $a=\bar{q}^{1 / 2}$, where $\bar{q}=\frac{1}{N} \sum_{i=1}^{N} q_{i}$ ( $N$ being the number of bases of the sequence). This value is around $a \simeq 0.07$, which is small enough to avoid spurious discretization effects when numerically integrating Eq. (2). In fact, taking Eq. (1) as an ansatz in Eq. (2) was a good choice, as the kink is a very robust object even in inhomogeneous sequences and its center can be well defined by interpolating the position where $\phi=\pi[18,19]$.

Once the model was defined, Salerno built a sequence $\left\{q_{i}\right\}$ to introduce it in (2). He was interested in the genomic sequence of the $T 7 A_{1}$ promoter but, instead of using the original DNA sequence, he built a "synthetic" one from the original. We will review all the details of this process as this will be the key to understanding the results of [13]. He took a sequence $S$ of 168 bases containing the so-called $A_{1}$ promoter (further details on $T 7$ promoters will be given in the next section) which 
corresponds to base pairs (BP) from 378 to 545 of the actual $T 7$ genomic sequence, and built a longer sequence of 1000 bases, that we will call $S^{\prime}$, to prevent the influence of boundary conditions on the dynamics of kinks:

$$
\begin{aligned}
S^{\prime}= & S(1,5)+8 S(1,50)+S(1,168) \\
& +15 S(141,168)+S(162,168) .
\end{aligned}
$$

In this way, the 168-base sequence $S$ would remain in the center of the new sequence $S^{\prime}$, with the transcription start site located in BP 526 and the promoter sequence going from BP 509 to BP 531, far from the limits of the lattice. Therefore, reflective boundary conditions could be safely used in the numerical simulations. We will return to this issue when discussing the results.

As it was known that the RNA polymerase could bind to DNA in the region of $S$ going from BP 51 to BP 140 (going from BP 455 to BP 545 in $S^{\prime}$ ), the expectation was that this region should be dynamically active. Hence, in [13] several integrations of Eq. (2) were carried out with the initial position of the static kink in a variety of sites inside the promoter region and the behaviour of the kinks was studied as a function of their starting position. The results were the following: For initial positions in $S^{\prime}$ from BP 415 to BP 505 the kink remained static or with small oscillations around the starting point. For BP 510, the kink acquired a velocity $v=0.18$ towards the left, was reflected without loss of energy at the left boundary and reflected again at the promoter region with velocity $v=$ 0.18 . This behaviour was enhanced when the initial position was increased from BP 510 to BP 535, where the kink also reached the maximum velocity $v=0.3$. Beyond this point this dynamical behaviour was drastically reduced. For BP 540 the kink acquired a small velocity $(v \simeq 0.08$ ) towards the right, and for BP 555 the kink simply remained at rest. The dynamics of a kink with initial velocity $v=0.3$ towards the left was also studied, starting from BP 900; it was found that the soliton was accelerated when it traveled from right to left through the central region, then reflected at the left end of the sequence and decelerated when traveled in the opposite direction. It was concluded in [13] that these results showed the existence of a dynamically "active" region going from BP 510 to BP 540 inside the $T 7 A_{1}$ promoter that could explain the functioning of DNA promoters as energetic activators of the RNA polymerase transport process.

In a subsequent paper, Salerno and Kivshar [15] introduced the effective potential in order to explain the behaviour of these objects when moving in an inhomogeneous sequence. The idea is that kink robustness allows us to approximate their dynamics, even though they are extended objects, as if they were point-like particles moving along a one-dimensional potential, given by

$V_{\mathrm{eff}}(n)=\frac{\sum_{m}\left(\bar{q}+q_{m}\right) \operatorname{sech}^{2}(a(m-n))}{2 \sum_{m} \operatorname{sech}^{2}(a(m-n))}$.

Recently this approach has been shown to give good results for Fibonacci [16] and DNA sequences other than the $T 7$ one [18, 19]. By "good results" we mean that the dynamics of the kink in

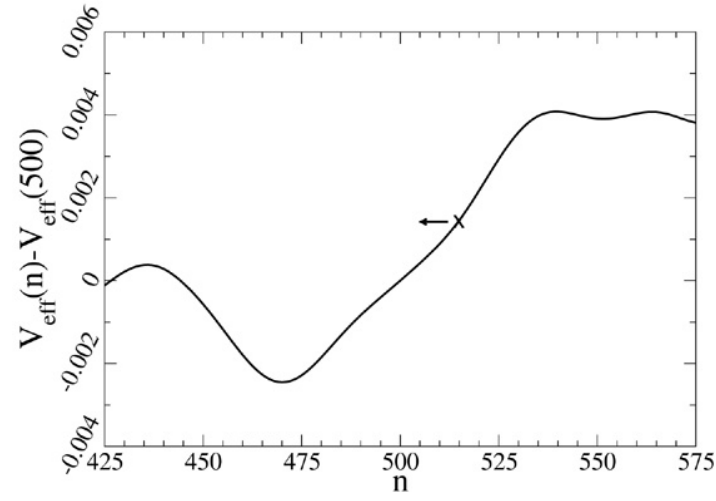

Fig. 1. Effective potential for $a=0.07$ of sequence $S^{\prime}$ from BP 425 to BP 605 . The only difference from the one represented in [15] is that, in the latter, an additional averaging of the potential over $\bar{q}^{-1 / 2}$ was carried out.

Eq. (2) and that of the particle in the effective potential (4) can by aligned, in the sense that trajectories are semiquantitatively similar, equilibrium points for the kink correspond to minima of the potential, and so on. This was also the case with the effective potential introduced in [15]: This paper reported the agreement of the direction of motion of the kinks according to the effective potential curve corresponding to the sequence $S^{\prime}$, plotted from BP 425 to BP 605 (see Fig. 1). As can be seen from the figure, there is indeed a good correspondence between the effective potential and the simulation results summarized above.

However, a more detailed analysis shows that this correspondence is not enough to establish a relation between DNA promoters and dynamically "active" regions. In Fig. 2 (a) there is plotted the effective potential $V_{\text {eff }}(n)$ (taking $V_{\text {eff }}(500)$ as the origin of energies) for sequence $S^{\prime}$ and for the original $T 7$ genome sequence. From this figure we immediately observe, on the one hand, that the positions of the peaks and the wells of the effective potential of sequence $S^{\prime}$ explain very well the above kink dynamics results reported in [13] in terms of a point-like particle; and, on the other hand, that the effective potential of the original $T 7$ genome far from the $A_{1}$ promoter is very different from that of the $S^{\prime}$ sequence, and hence the dynamics of kinks must be different, too. Fig. 2 (b) shows the dynamics of two kinks on the two sequences, $S^{\prime}$ and the original $T 7$ sequence. It is clear that the dynamics behaviours of the two sequences are greatly different: for instance, in the true potential BP 510 should not be regarded as an active site whereas BP 680 should be regarded so. The comparison of the trajectories with those obtained from the effective potential confirms the validity of this potential for describing the dynamics of kinks (allowing for a difference in time scales, as in the point-like particle description time units are arbitrary). This means that the effective potential is a correct description for both the real sequence and $S^{\prime}$, and therefore the differences between them are not an artifact of this approximation. These differences between the two potentials come from the periodic sequences introduced in (3), $8 S(1,50)$ and $15 S(141,168)$, adjacent to the 168-nucleotide $S$ sequence. The AT/CG content in the periodic sequences has an average value around which the effective potential of these sites oscillates. As further evidence of the influence of the ends of the sequence, in Fig. 3 we show 
(a)

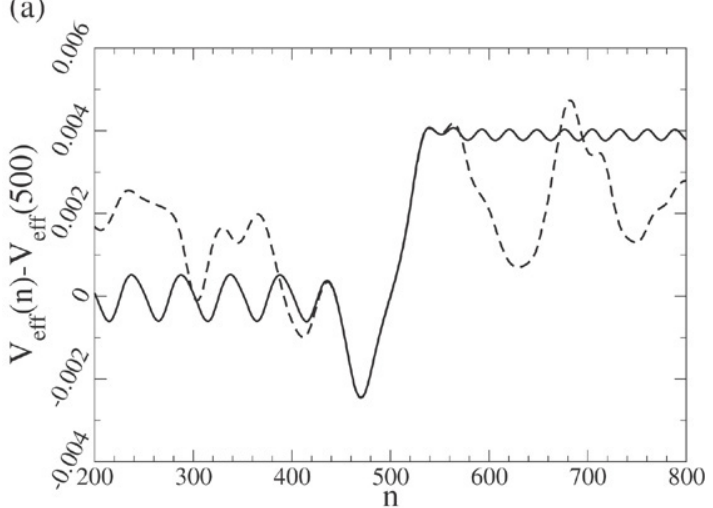

(b)

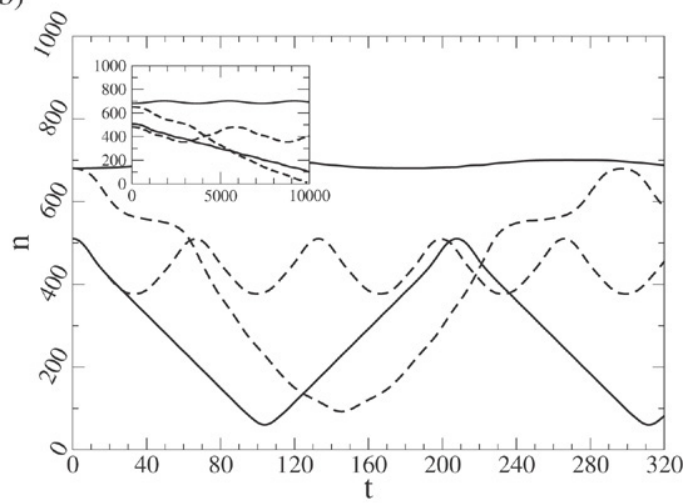

Fig. 2. (a) Effective potential for $a=0.07$ around the $T 7 A_{1}$ promoter for the synthetic sequence $S^{\prime}$ (solid line) and for the original genome sequence (dashed line). The potential of the original sequence has been shifted along the horizontal and vertical axes to make it coincide with that of the 168-base sequence $S$ in $S^{\prime}$. (b) Dynamics of the center of two kinks (calculated by interpolating the position where $\phi=\pi$ ), moving along the $S^{\prime}$ sequence (solid lines) and the real $T 7$ genome sequence (dashed lines), starting from BP 510 and BP 680. Inset: point-like particle dynamics starting from the same sites and moving according to the corresponding effective potentials of (a). Although the dynamics is scaled in time with respect to the kink dynamics, the trajectories of each pair particle/kink are the same.

Table 1

Summary of the dynamical results for kinks moving in the synthetic sequences $S^{\prime}$ obtained from $A_{1}, A_{0}$ (also called $D$ ) and $A_{3}$ promoters in [14]

\begin{tabular}{|c|c|c|}
\hline Promoter & $\mathrm{BP}$ region & Response \\
\hline$A_{1}$ & $\begin{array}{l}\text { From } 510 \text { to } 535 \\
540\end{array}$ & $\begin{array}{l}\text { Leftward propagation, strongest at } 535 \text {. } \\
\text { Small velocity towards the right. }\end{array}$ \\
\hline$A_{0}($ or $D)$ & $\begin{array}{l}\text { From } 530 \text { to } 540 \\
\text { From } 543 \text { to } 555\end{array}$ & $\begin{array}{l}\text { Leftward propagation, strongest at } 540 . \\
\text { Rightward propagation, strongest at } 543 \text {. }\end{array}$ \\
\hline$A_{3}$ & From 435 to 460 & Rightward propagation. \\
\hline
\end{tabular}

the effective potential of sequences $S^{\prime}, S_{1}^{\prime}$ and $S_{2}^{\prime}$, with $S_{1}^{\prime}=$ $405 A+S(1,168)+427 A$ and $S_{2}^{\prime}=405 C+S(1,168)+427 C$, where $N A(N C)$ means $N$ consecutive sites with nucleotide $A(C)$. The effective potentials for kinks moving along $S_{1}^{\prime}$ and $S_{2}^{\prime}$ will lead to a dynamics very different from that described in [13] and reported here, although they all have the same central sequence of 168 nucleotides.

In [14] the same methodology as was developed in [13] was used to analyze another two $T 7$ promoters, namely $A_{0}$

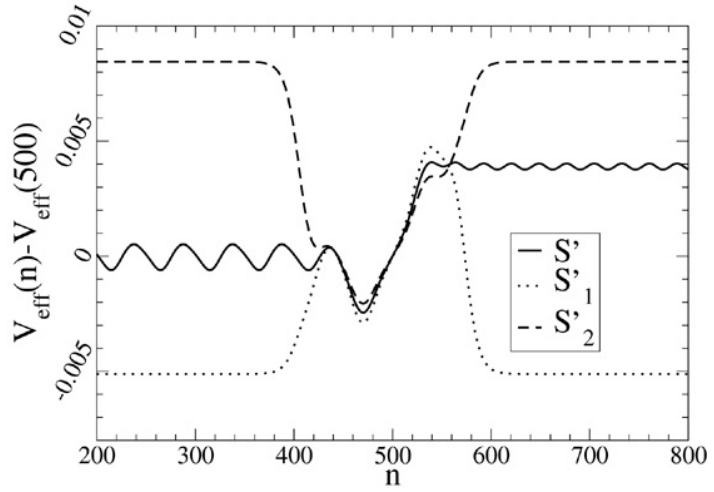

Fig. 3. Relevance of the end parts of the sequence: Effective potential for the synthetic sequences $S^{\prime}, S^{\prime}{ }_{1}$ and $S_{2}^{\prime}$ (see the text for definitions).

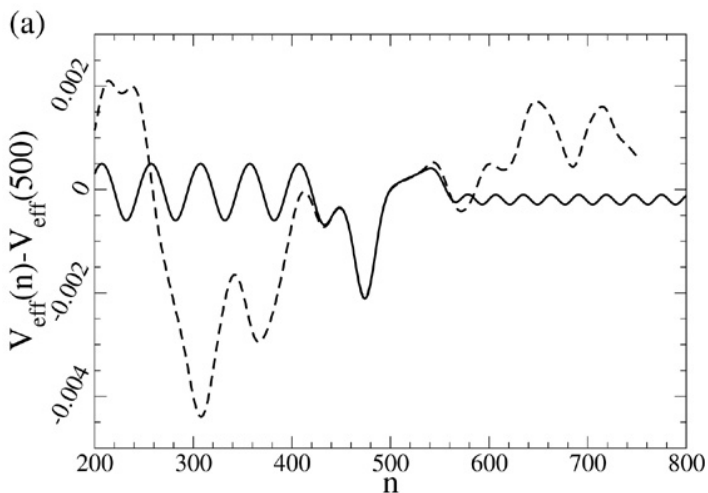

(b)

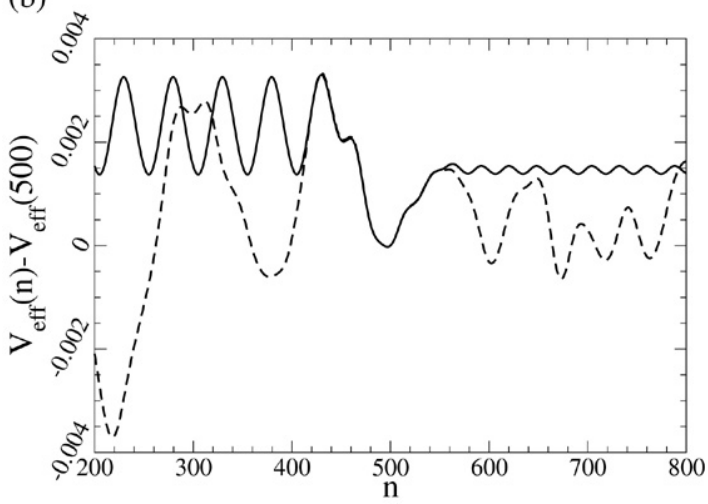

Fig. 4. (a) Effective potentials for $a=0.07$ of the synthetic sequence $S^{\prime}$ (solid line) and for the original genome sequence (dashed line) around the $T 7 A_{0}$ (also called $D$ ) promoter. The sequence used in [14] corresponds to the transcription strand (this promoter activates transcription leftwards, and therefore it uses the complementary strand of the sequence usually shown [21, 22]) in the transcription order. Therefore, the original sequence has been written in reverse order in order to obtain its potential, and then shifted along the horizontal and vertical axes to make it coincide with the potential of the $S$ sequence in the synthetic $S^{\prime}$ sequence. (b) Effective potentials for $a=0.07$ of the synthetic sequence $S^{\prime}$ (solid line) and for the original genome sequence (dashed line) around the $T 7 A_{3}$ promoter. The potential of the original $T 7$ sequence has been shifted in order to make it coincide with the potential of the $S$ sequence in the synthetic $S^{\prime}$ sequence.

(also called $D$ ) and $A_{3}$, and similar results were obtained (see Table 1). Figs. 4(a) and (b) show the effective potential of the 
synthetic sequences built by Salerno from the genomic 168nucleotide sequences and the effective potentials of the real $T 7$ sequences around the promoters. Again, the effective potentials of the synthetic sequences describe the results of the dynamics summarized in Table 1, but differ from the effective potentials of the real genomic sequences, yielding different dynamics. For instance, according to Fig. 4 (a), a kink starting from BP 245 in the real genomic sequence around the $A_{0}$ promoter would reach the right end of the sequence, instead of oscillating around the initial starting position, as it would do in the corresponding $S^{\prime}$ sequence.

We note that in [14] it was argued that, as the initial static soliton was always well inside the original 168-base sequences, then the flanking regions used to extend the chain played no role in the dynamical effects described. However, we have just shown how important they are when the kink moves towards them. Therefore, we are forced to conclude that the results in $[13,14]$ are highly dependent on the construction of sequences $S^{\prime}$, and that when the original $T 7$ genome sequence is used instead then the promoter regions cannot be considered "active" or "special" regions any longer. As we have seen, other regions close to, but different from, the promoters may be even more "active" in the sense of inducing kink motion; conversely, some active regions in the synthetic sequence lose this character in the real genome.

\section{Subsequent developments: Full $T 7$ genome}

Following the interesting proposal of Salerno, namely the putative relation between $T 7$ promoters and the dynamics of solitons moving along inhomogeneous genomic sequences, further research intended to shed more light on this question [17]. The main contribution of this sequel is that the sequences used were real genomic sequences and, in addition, that the whole $T 7$ genome was studied.

In the research reported in [17], Lennholm and Hörnquist measured the maximum distance (in either direction) reached by initially static kinks starting from each of the sites of the whole sequence of the $T 7$ genome. They also took the 24 promoters of the $T 7$ genome (except the first and the last ones to avoid boundary effects), studied the results obtained for positions going from -4 to -1 of each promoter and compared these results with the results of the whole genome. The aim of this analysis was to find whether the RNA polymerase melting region (the one going from -4 to -1 in each promoter) acts as a dynamically "active" region as proposed by Salerno, or behaves in the same way as the rest of the nucleotides of the genome. In this respect, they did not find relevant differences (see Fig. 1 of [17]). However, for every promoter they investigated the activity of the first $n$ base pairs which are transcribed by RNA polymerase and found that, for $n=20$, the regions studied are more active than average with a significance of more than five standard deviations (see Fig. 2 of [17]). They did not give any biological interpretation of that results but, in their conclusions, they suggested that a more quantitative relation between kink motion and the effective potential should be established.
As already mentioned, this paper as well as our previous work $[16,18,19]$ has proven the agreement between kink dynamics and effective potential. Therefore, we can now study the whole $T 7$ genome in terms of this tool. To this end, we will review some of the properties of the $T 7$ promoters in order to set a methodology in the study of the effective potential in these regions. The $T 7$ phage genome is one of the most studied genomes since the whole genome sequence was found in 1983 [21], and few changes in the sequence have been reported since then [22]. The reproductive cycle of the $T 7$ phage is closely linked to the promoter and gene distribution in the genome. When the T7 RNA is injected inside a bacteria, like E. coli, the bacterial RNA polymerase starts to produce mRNAs induced by three major promoters from the early region (or class I region) $A_{1}, A_{2}$ and $A_{3}$. A fourth major $E$. coli promoter, $A_{0}$ (also called $D$ ), that would direct transcription leftward, and several minor E. coli promoters function in vitro but have no known in vivo function. Once the $T 7$ phage has its own transcription machinery, late mRNAs are produced by 15 promoters for $T 7$ RNA polymerase distributed across the rightmost $85 \%$ of the DNA (divided into class II and class III regions). There are also two $T 7$ promoters associated with possible origins of replication at the left and right ends of $T 7$ DNA. The 23-base-pair consensus sequence for $T 7$ RNA polymerase promoters stretches from -17 to +6 , where +1 is the transcription start site. For E. coli RNA polymerase, the consensus sequence is formed by two hexamers around positions -10 and -35 from the transcription start site. This means that the nucleotides of the promoters are highly correlated at these sites (although sometimes they are not strictly the same), but not in the rest of the sequence (we will come back to consensus sequences below).

We can now go back to our main aim: We want to find out whether or not there is some kind of pattern in the effective potential, or a set of properties to be applied to all the promoters in the $T 7$ genome that allow their identification among the rest of the genome. To this end, we must keep in mind that the effective potential on each site (see Eq. (4)) is just a weighted average of the sequence around the site, with weight function $\operatorname{sech}^{2}(a n)$. We can obtain an estimation of the resolution of the effective potential reading frame by noticing that an error of about $10 \%$ in the effective potential is introduced when truncating the sum in the weighted average (4) in $\pm \Delta n=$ $1.5 a^{-1}$ around each $n$. If we consider that, for further sites, the contribution of the $q_{m}$ to the weighted average is negligible, then the number of sites averaged when computing the effective potential on each site goes as $\Delta n \simeq 3 a^{-1}$. This means that, for $a \simeq 0.07$ (which is the approximate value of the discretization as explained in Section 2), $\Delta n$ is about 40, a much lower resolution than the one needed to recognize a 23base-pair consensus sequence in the effective potential (and even worse for recognizing hexamers). Therefore, we conclude that the kink is too wide to allow us to check that the same curve describes the effective potential of different promoters, as was suggested in $[13,14,17]$. On the other hand, we can increase the resolution of the effective potential by increasing the discretization $a$ until reaching $\Delta n=1$. We could find 
(a)

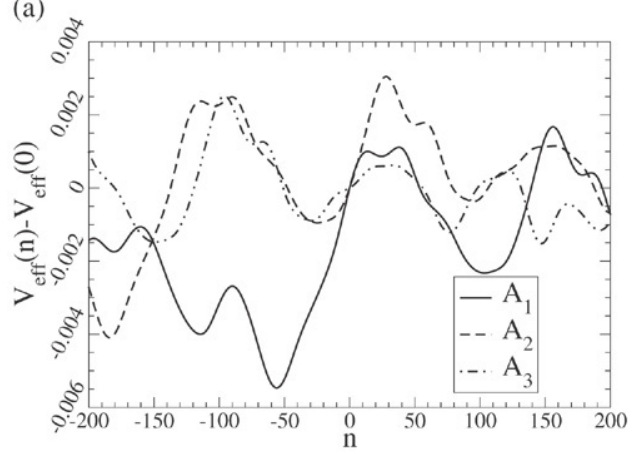

(c)

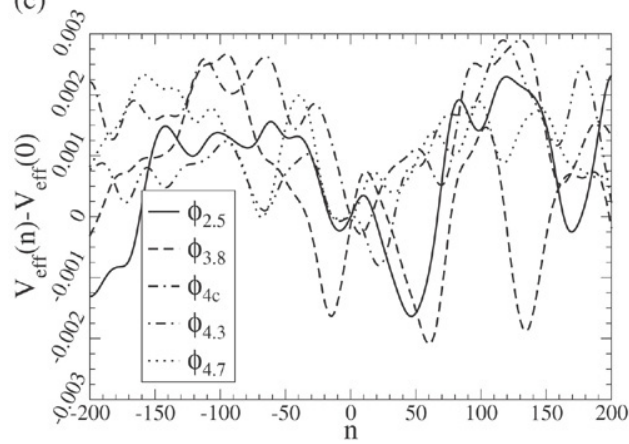

(b)

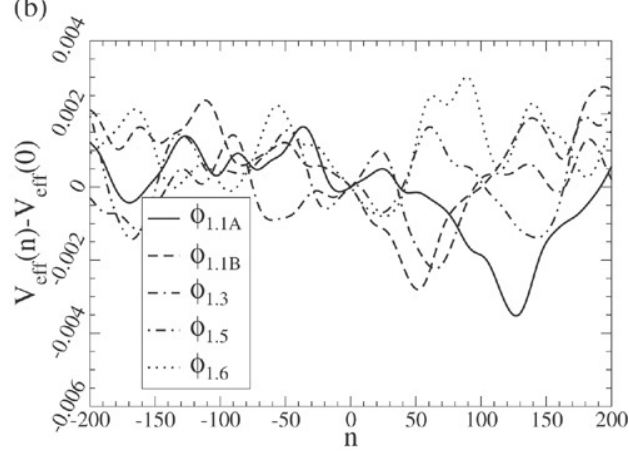

(d)

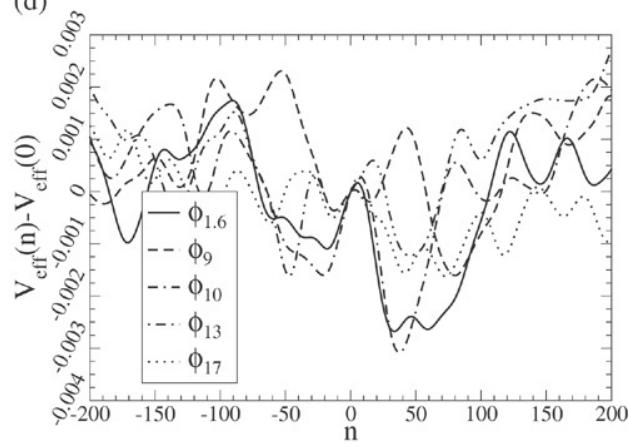

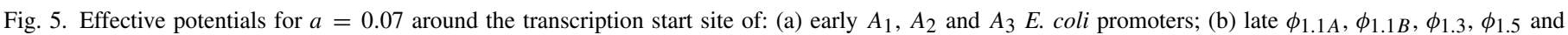

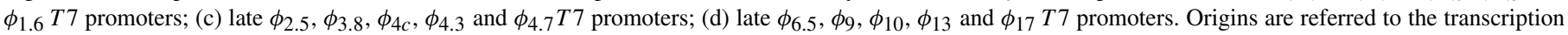
start site in all cases.

then the consensus sequence repeated in the effective potential around each promoter, but that would not give more information than the consensus sequence itself, and the effective potential would not be useful from a genomic point of view.

To go beyond the previous theoretical discussion, we have computed the effective potential for most of the $T 7$ promoters for $a=0.07$. In Fig. 5 we show the effective potential of the three major $E$. coli RNA polymerase promoters (early promoters) and the $T 7$ RNA polymerase promoters (late promoters) of $T 7$ for $a=0.07$. Clearly there is no "consensus effective potential" that appears in all (or in most) of the promoters. If we were looking for more subtle properties that might enclose all the 18 promoters or each subset of early and late promoters, then we would be led to consider as promoters other regions of the $T 7$ genome which are not promoters. As an example, the effective potentials around some other regions that are not promoters are plotted in Fig. 6, and it is shown how alike they look to the ones of Fig. 5. Hence, we believe that the effective potential of kinks, and therefore the dynamics of kinks in the inhomogeneous $\mathrm{sG}$ model, cannot explain the initiation in the promoters of the transcription process in the $T 7$ phage and, probably, in any other organism.

We now turn to the work in [17] in order to understand the difference from the conclusions reported there. The research in [17] is certainly interesting because, as we already said, it is the first time that the whole genomic sequence of the $T 7$ phage was taken into account. However, we believe that their methodology is not appropriate for the case under study, as the statistical analysis of kink dynamics does not give conclusive

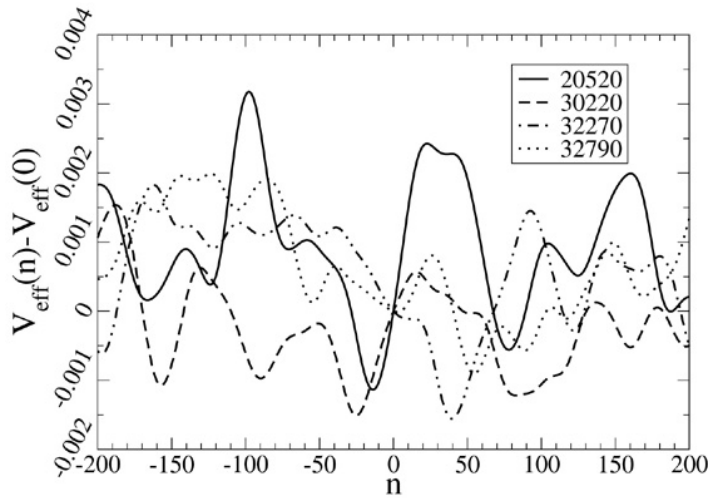

Fig. 6. Effective potential for $a=0.07$ at different sites of the sequence, which may look like the effective potential of promoters in Fig. 5 but which are not. The origin of each sequence corresponds to the number in the legend box.

results. For instance, a graph with the furthest position reached in the sequence in terms of the initial position from which the kink started to move would have yielded different results from the ones reported in $[13,14]$ and the work would have been more conclusive. In addition, we note that the direction of motion of the kinks was not recorded and therefore it cannot be assessed whether or not the "activity" of those regions agrees with the transcription direction. We therefore conclude that an individual study of each promoter is needed if functionally relevant places are to be found. This individual study is what we have presented here and we believe that the conclusion is clear: The effective 
potential shows no signature of the promoters. Having verified this, in the next section we show that, if a detailed study of promoters is made, it must be over all the promoters of the phage in order to be conclusive.

\section{4. sG breathers}

In this last section, we consider yet another recent approach to sG soliton dynamics that followed the steps of [13-15] but using breathers instead of kinks [20]. In this case, the author studied RNA polymerase recognition of specific binding sites by comparing breathers to localized deformations of the DNA duplex when the RNA polymerase slides on its major groove. To this end he constructed a potential for $\mathrm{sG}$ breathers following the steps of [15], but using as ansatz a discretized breather, given by

$\phi_{\mathrm{br}, n}(t)=4 \tan ^{-1}\left(\tan \mu \frac{\sin \left(t \bar{q}^{1 / 2} \cos \mu\right)}{\cosh \left(n \bar{q}^{1 / 2} \sin \mu\right)}\right)$,

where $\mu$ is related to the intrinsic frequency of the breather. The potential obtained in [20] is the following:

$V_{\mathrm{br}}(n)=4 \tan ^{2} \mu \sum_{m} \frac{\left(\bar{q}+q_{m}\right) \cosh (a(m-n))}{\left(\tan ^{2} \mu+\cosh ^{2}(a(m-n))\right)^{3 / 2}}$,

with $a=\bar{q}^{1 / 2} \sin \mu \simeq 0.04$. The main difference between this potential and the one obtained in [15] is that breathers defined by (5) are not static solutions of the sG model. This means, on the one hand, that the kinetic term of the $\mathrm{sG}$ Hamiltonian (that we do not write here) has two extra terms when deriving $\phi_{\mathrm{br}, n-n_{0}(t)}$ with respect to time and squaring it, and that the potential term obtained depends explicitly on time. These problems may be solved by moving one of the extra terms from the kinetic to the potential term, and then integrating in time over a period. Another important difference from [15] is that kinks are very robust objects that behave very well in the discrete $\mathrm{sG}$ model, even for inhomogeneous sequences, and that is why they can be expressed in terms of its center. Breathers, however, are very unstable in the homogeneous, discrete sG model, and it is to be expected that they are even more so on inhomogeneous sequences. Therefore, we do not think that the potential for breathers may describe accurately and/or for long times the dynamics of an initially static breather. The construction of the $\mathrm{sG}$ potential for breathers is, therefore, not as straightforward as the one for kinks in [15], and this must be taken into account when analyzing the results.

After the breather potential (6) was constructed in [20], it was used to analyze the early region of the $T 7$ genome and a particular region of the $T 5$ phage. It was suggested, among other things, that there is a correlation between deep wells of (6) and promoters in the early region and class III $T 7$ promoters. Another relation between deep wells and transcription terminators was also suggested. We can now apply the results obtained in Section 3 to the claims in [20] by noticing that the weight functions of (4) and (6) look very alike for $\tan \mu<1$ (which is the case, according to [20]). Therefore, the structures of peaks and wells are very similar

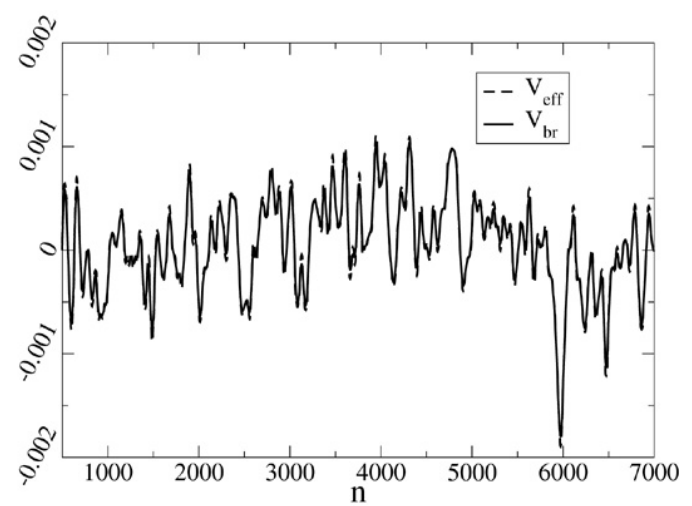

Fig. 7. Potential $V_{\text {br }}$ (6) for breathers (solid line) and $V_{\text {eff }}$ (4) for kinks (dashed line) for the $T 7$ genome region going from BP 500 to BP 7000 for $a=0.04$ and $\mu=\pi / 6$ (which corresponds to the values used in the figures of [20]). The scale of $V_{\mathrm{br}}$ has been divided by 50 in order to make it fit with the scale of $V_{\mathrm{eff}}$. No vertical shifting was carried out for any potential. This region of the genome contains seven promoters.

in the two cases, as shown in Fig. 7, and we can thus extend the conclusions of Section 3: Even if the potential for sG breathers works in a similar way to the effective potential for sG kinks, it is not enough to explain the transcription process of RNA polymerase. For instance, as shown in [20], there are deep wells in the potential for $\mathrm{sG}$ breathers near some of the promoters of the $T 7$ genome. However, there are other promoters (class II) which are not near any deep well, and also deep wells which are not near promoters (like the ones found in [20] near terminators). When losing the constraints in order to take into account not so deep wells which are near promoters, then many other wells far from promoters should be considered, too. We therefore conclude that there is no special characteristic in the potential for breathers that allows the identification of promoters from the rest of the genome, by simply looking at the effective potential.

\section{Conclusions}

The Englander model was introduced in [7] to explain long lifetimes on open states of DNA duplexes [10] by means of the well known nonlinear sG model. Subsequently, research on the sG model led to suggestions of a relation between functionally relevant positions in the sequence with dynamical properties of sG solitons. In Section 2 we showed that the results of kink dynamics moving along inhomogeneous sequences developed in $[13,14]$ depend greatly on the sequence under study. In order to achieve this we used the effective potential for $\mathrm{sG}$ kinks moving on inhomogeneous sequences, introduced in [15]. Applying this to the sequences used in $[13,14]$ and to the corresponding real genomic sequences of the $T 7$ phage, we observed important differences between the two potentials. Differences came from the end parts of the analyzed sequences, which were a priori assumed not to have any role. With these findings and taking into account the good results already obtained for the particle-like approximation of sG kinks moving along inhomogeneous sequences $[16,18,19]$, we concluded that 
early promoter regions of the $T 7$ genome cannot be considered dynamically "active". In Section 3, addressing the question posed in [17], we searched for patterns that could differentiate the dynamics of kinks starting from $T 7$ promoters from kinks starting from the rest of the genomic sequence. Again, we used the effective potential, this time applied to the whole genomic sequence of the $T 7$ phage. Comparing the curves obtained for the 18 major promoters of the phage among them and also with other non-promoter regions led us to think that there were no special properties of the effective potential around promoter regions, and therefore that the dynamics of kinks moving from these regions was the same as in other genomic regions. Finally, in Section 4 we applied the same arguments and also reviewed the problems of the potential for breathers in order to demonstrate that the potential for $\mathrm{sG}$ breathers obtained in [20] cannot be used to differentiate promoter regions in the genomic sequence. From all this evidence, we can confidently claim that neither the $\mathrm{sG}$ model nor its description in terms of the effective potential give hints about functionally relevant sites of DNA sequences. We stress that this claim concerns the sG model and the dynamics of its solitons. Statistical mechanics approaches are also being studied with some success [26,2325 ] but that is a completely different approach.

It is important to extend this discussion to include its biological implications. The relation of deep wells and functioning sites of DNA can now be discussed in terms of properties of bacterial promoters [27,28]. Bacterial RNA polymerase is a multisubunit complex. A detachable subunit, called a $\sigma$ factor, is responsible for reading the promoters, which are the signals encoded in the DNA that tell it where to begin transcribing. Most bacteria contain multiple $\sigma$ factors that enable the recognition of different sets of promoters. A comparison of many different bacterial promoters reveals that they are heterogeneous in the DNA sequence. However, they all contain related sequences that are reflected in mechanical and electrostatic properties of the DNA double helix that are recognized by the $\sigma$ factor. These common features are often summarized in the form of a consensus sequence, which serves as a summary or "average" of a large number of individual nucleotide sequences. The precise sequence determines the strength (or number of initiation events per unit time) of each promoter. However, although the $\sigma$ factor is needed in the transcription initiation, other elements can bind to RNA polymerase to regulate the transcription of specific promoters, like the $\alpha$ subunits. Another important group of proteins that recognize and bind to promoters are the transcription factors. These proteins act as regulatory elements that control transcription initiation and bind to specific sequences. This summary of regulatory elements of transcription initiation in prokaryotes reveals the intrinsic complexity of the sequences of promoters. In the case of eukaryotic regulation the complexity increases too much to try to summarize it in this paragraph, and we will just refer to the counterintuitive fact that specific CG-rich promoters that are found in yeast [29]. Therefore, we conclude that, although deep wells in the potential for $\mathrm{sG}$ kinks or breathers are correlated with AT-rich regions, they are not enough for recognizing such complex structures as promoters, and it is only natural that the dynamics of these simple excitations cannot capture the mechanisms of promoter function.

\section{Acknowledgements}

We thank Antonio Marín for patient and helpful discussions about promoters. This work was supported by the Ministerio de Educación y Ciencia of Spain through grants BFM2003-07749C05-01, FIS2004-01001, NAN2004-09087-C03-03, FIS2005973, by Comunidad de Madrid grant SIMUMAT-CM and by the Junta de Andalucía under projects 00481 and FQM-0207. S.C. is supported by a fellowship from the Consejería de Educación de la Comunidad de Madrid and the Fondo Social Europeo.

This work originated from discussions at a Summer School in 2005 at Baeza, Spain. We are grateful to Renato Álvarez Nodarse for creating that nice atmosphere.

\section{References}

[1] E. Fermi, J.R. Pasta, S. Ulam, Los Alamos Report LA-UR-1940 (1955); E. Segré (Ed.) Collected Papers of Enrico Fermi. University of Chicago, Chicago, 1965 (Reprint).

[2] A.S. Davydov, Solitons in Molecular Systems, second ed., Kluwer, Dordrecht, 1991.

[3] L.V. Yakushevich, Nonlinear Physics of DNA, second ed., Wiley, Chichester, 2004.

[4] G. Gaeta, J. Biol. Phys. 24 (1999) 81.

[5] M. Peyrard, A.R. Bishop, Phys. Rev. Lett. 62 (1989) 2755.

[6] M. Peyrard, Nonlinearity 17 (2004) R1.

[7] S.W. Englander, N.R. Hippel, A.J. Heeger, J.A. Krumhansl, A. Litwin, Proc. Natl. Acad. Sci. USA 77 (1980) 7222.

[8] J. Maddox, Nature 324 (1986) 11

[9] M. Frank-Kamenenskii, Nature 328 (1987) 108.

[10] J.J. Englander, P.H. von Hippel, J. Mol. Biol. 63 (1972) 171.

[11] M. Guéron, M. Kochoyan, J.-L. Leroy, Nature 328 (1987) 89.

[12] A very illustrative explanation of the effect of water friction inside cells can be found in chapter $5 \mathrm{P}$. Nelson, Life in the slow lane: The low Reynolds-number world, in: Biological Physics: Energy, Information, Life, W. H. Freeman \& Co., New York, 2004.

[13] M. Salerno, Phys. Rev. A 44 (1991) 5292.

[14] M. Salerno, Phys. Lett. A 167 (1992) 49.

[15] M. Salerno, Y.S. Kivshar, Phys. Lett. A 193 (1994) 263.

[16] F. Domínguez-Adame, A. Sánchez, Y.S. Kivshar, Phys. Rev. E 52 (1995) R2183.

[17] E. Lennholm, M. Hörnquist, Physica D 177 (2003) 233.

[18] S. Cuenda, A. Sánchez, Fluct. Noise Lett. 4 (2004) L 491.

[19] S. Cuenda, A. Sánchez, Phys. Rev. E 70 (2004) 51903.

[20] J.D. Bashford, J. Biol. Phys. 32 (2006) 27.

[21] J.J. Dunn, F.W. Studier, J. Mol. Biol. 166 (1983) 477.

[22] http://www.ncbi.nlm.nih.gov.

[23] C.H. Choi, G. Kalosakas, K.Ø. Rasmussen, M. Hiramura, A.R. Bishop, A. Usheva, Nucleic Acid. Res. 32 (2004) 1584.

[24] Z. Rapti, A. Smetzi, K.Ø. Rasmussen, A.R. Bishop, C.H. Choi, A. Usheva, Europhys. Lett. 74 (2006) 540.

[25] T. van Erp, S. Cuesta-López, J.-G. Hagmann, M. Peyrard, Phys. Rev. Lett. 95 (2005) 218104.

[26] C.H. Choi, A. Usheva, G. Kalosakas, K.Ø. Rasmussen, A.R. Bishop, Phys. Rev. Lett. 96 (2006) 239801;

T. van Erp, S. Cuesta-López, J.-G. Hagmann, M. Peyrard, Phys. Rev. Lett. 96 (2006) 239802.

[27] B. Alberts, A. Johnson, J. Lewis, M. Raff, K. Roberts, P. Walter, Molecular Biology of the Cell, Garland Publishing, 2002.

[28] D.F. Browning, S.J.W. Busby, Nature Rev. Microbiol. 2 (2004) 1.

[29] A. Marín, M. Wang, G. Gutiérrez, Gene 333 (2004) 151. 